\title{
Niveau De Connaissance De La Ménopause Et Habitudes Alimentaire Et Médicinale Des Femmes En Côte d'Ivoire
}

\author{
Kouame Amoin Gervaise, Doctorante \\ Koffi Yao Mesmin, Doctorant \\ Piba Serge Cherry, Docteur \\ UFR Sciences de la Nature, \\ Université Nangui Abrogoua, Abidjan, Côte d'Ivoire. \\ Bakayoko Adama, Professeur
}

UFR Sciences de la Nature, Université Nangui Abrogoua, Abidjan, Côte d'Ivoire/ Centre Suisse de Recherches Scientifiques en Côte d'Ivoire, Abidjan, Côte d'Ivoire.

Tra Bi Fézan Honora, Professeur

UFR Sciences de la Nature, Université

Nangui Abrogoua, Abidjan, Côte d'Ivoire.

KONE Mamidou Witabouna, Maître de Conférences

UFR Sciences de la Nature, Université Nangui Abrogoua, Abidjan, Côte d'Ivoire/ Centre Suisse de Recherches Scientifiques en Côte d'Ivoire, Abidjan, Côte d'Ivoire.

Doi: 10.19044/esj.2018.v14n18p442 URL:http://dx.doi.org/10.19044/esj.2018.v14n18p442

\begin{abstract}
Menopause is accompanied by symptoms that are difficult for women to tolerate. In Asia, plants used by some women allow them to live well in menopause. The purpose of this study was to assess women's knowledge level of menopause and the diversity of local medicinal and food plants that they are accustomed to before this stage. A survey was conducted in the departments of Grand Lahou and Katiola with 267 women aged 40 to 70 using semistructured interviews. The results showed that the average age of menopause was $48.88 \pm 4.19$ years. In total, $96.85 \%$ of women considered that menopause is normal from the age of 50 and has no effect on health. For $90.94 \%$ of them, stopping menstruation during menopause is perceived as a release but difficult to withstand because of the disorders felt. The symptoms and risks of menopause were unknown respectively by $73.68 \%$ and $98.71 \%$ of women. Hot flashes were the best known. A variety of medicinal and food plants (47) are used by postmenopausal women who have no symptoms or very few
\end{abstract}


symptoms of menopause. These plants may have an interest in controlling the symptoms of menopause.

Keywords: Menopause, Medicinal plants, Food plants, Phytoestrogens, Ivory Coast

\section{Resume}

La ménopause s'accompagne de symptômes difficilement supportables par les femmes. En Asie, des plantes consommées par certaines femmes leur permettent de bien vivre leur ménopause. L'objectif de ce travail a été d'évaluer le niveau de connaissance des femmes de la ménopause et la diversité des plantes médicinales et alimentaires locales entrant dans leur habitude avant cette étape. Une enquête a été menée dans les départements de Grand-Lahou et de Katiola auprès de 267 femmes âgées de 40 à 70 ans à l'aide d'entretiens semi-structurés. Les résultats ont montré que l'âge moyen de la ménopause est de 48,88 \pm 4,19 ans. Au total 96,85\% des femmes ont estimé que la ménopause est normale à partir de 50 ans et sans conséquence sur la santé. Pour 90,94 \% d'entre elles, l'arrêt des menstrues pendant la ménopause est perçu comme une libération mais difficilement supportable à cause des troubles ressentis. Les symptômes et les risques de la ménopause sont méconnus respectivement par 73,68 \% et 98,71\% des femmes. Les bouffées de chaleur sont les plus connues. Une diversité de 47 plantes médicinales et alimentaires est utilisée par les femmes ménopausées ne présentant pas de symptômes ou présentant très peu de symptômes de la ménopause. Ces plantes pourraient avoir un intérêt dans le contrôle des symptômes de la ménopause.

Mots-clé : Ménopause, Plantes médicinales, Plantes alimentaires, Phytoestrogènes, Côte d'Ivoire

\section{Introduction}

La ménopause est un processus physiologique normal qui marque la fin des menstrues. Les changements hormonaux pendant la ménopause (climatère ou retour d'âge) sont associés à de nombreux symptômes physiques et psychologiques avec un risque accru de plusieurs maladies chroniques, dont l'ostéoporose, les maladies cardiovasculaires et le cancer (Al-Eassa et al., 2012). Les symptômes qui accompagnent généralement la ménopause sont gênants et peuvent, dans certains cas, réduire la mobilité ou l'activité socioprofessionnelle de la femme. Ce sont les bouffées de chaleur, les douleurs articulaires et musculaires, les céphalées, les palpitations et les insomnies (Lopès et al., 2004 ; Luoto, 2009). Selon Lopès et al., (2004), les bouffées de chaleur sont les signes qui caractérisent, le mieux, l'entrée en ménopause car elles apparaissent chez près de $50 \%$ des femmes. Certaines femmes $(1 \%)$ 
n'auront aucun symptôme durant la ménopause alors que celles qui en ressentent (99\%) (Loukid et al., 2007), ont leur qualité de vie grandement affectée. Les femmes passent une période appréciable de leur vie en postménopause car elle intervient généralement entre 45 et 55 ans dans le monde et entre 48 et 49 ans en Côte d'Ivoire (MSLS et INS et ICF International, 2013). Si les effets de la ménopause sur l'organisme de la femme sont bien connus dans les pays développés, la situation est contraire en Afrique. Peu de travaux sont consacrés à la question.

La médecine moderne offre des traitements hormonaux de la ménopause (THM) pour faire face aux malaises (Palacios, 2008 ; Bumbu et al., 2016). Ce traitement a l'avantage d'éliminer de nombreux symptômes, les risques de maladies cardiovasculaires et les fractures ostéoporotiques. Cependant, il présente de nombreux effets nocifs en provoquant le développement du cancer du sein (Drapier-Faure, 2003), des accidents thromboemboliques et vasculaires cérébraux, avec une prévalence de 2,3\% par an (Henri, 2005). Par ailleurs, les THM ne sont pas accessibles et disponibles, dans bon nombre de pays Africains et particulièrement en Côte d'Ivoire. Aujourd'hui, les femmes se tournent de plus en plus vers les plantes médicinales et alimentaires en raison de la présence des phytoestrogènes dont l'efficacité est prouvée (Delia et al., 2017). Il est montré que les femmes asiatiques ayant une alimentation traditionnelle basée sur le soja vivaient mieux leur ménopause que les femmes occidentales (Amiot et al., 2012). L'efficacité de la phytothérapie et de ses bienfaits incontestables pour la santé ont permis à la médecine naturelle d'entrer dans les habitudes quotidiennes des populations, au point où les pays les plus développés ne sont pas en reste (Pastor, 2006). En Côte d'Ivoire comme dans plusieurs pays africains, l'usage des plantes fait partie intégrante des habitudes alimentaires et médicinales des femmes. Les femmes africaines ont recours aux plantes médicinales et aux légumes feuilles en fonction de leur disponibilité sur les marchés ou dans les formations végétales. C'est ainsi que ce travail a été centré sur l'étude ethnobotanique des plantes médicinales et alimentaires locales entrant dans les habitudes des femmes âgées de 40 à 70 ans des départements de Grand-Lahou et de Katiola. L'objectif de ce travail est d'évaluer le niveau de connaissance des femmes de la ménopause et la diversité des plantes qui pourraient les aider à mieux vivre cet état.

\section{Matériel et méthodes \\ Zone d'étude}

L'étude s'est déroulée dans les départements de Grand-Lahou et de Katiola respectivement au Sud et au Centre Nord de la Côte d'Ivoire (Figure 1). Le département de Grand-Lahou est situé dans la zone de forêt sempervirente entre les latitudes $5^{\circ} 12^{\prime}$ et $5^{\circ} 9^{\prime}$ et les longitudes $4^{\circ} 56$ et $5^{\circ} 70$. 
La population du département de Grand-Lahou est estimée à 151313 d'habitants avec 71019 femmes (INS, 2015). Plusieurs ethnies vivent dans ce département. Il s'agit des Avikams, des Didas et des N'Zima qui représentent les autochtones, des Agnis, Baoulés, Malinkés, et Gouros pour les allochtones, et des allogènes Ghanéens, Maliens et Burkinabés.

Le département de Katiola est situé, dans la zone Subsoudanaise, entre $8^{\circ} 10^{\prime}$ de latitude nord et $5^{\circ} 4^{\prime}$ de longitude ouest. La population est de 106905 habitants dont 51635 femmes (INS, 2015). Quatre (4) grands groupes ethniques peuplent ce département. Ce sont majoritairement les Tagbana et les Mangôrô suivis des allogènes Sénoufos et Baoulés de même que des communautés Maliennes et de Burkinabés.

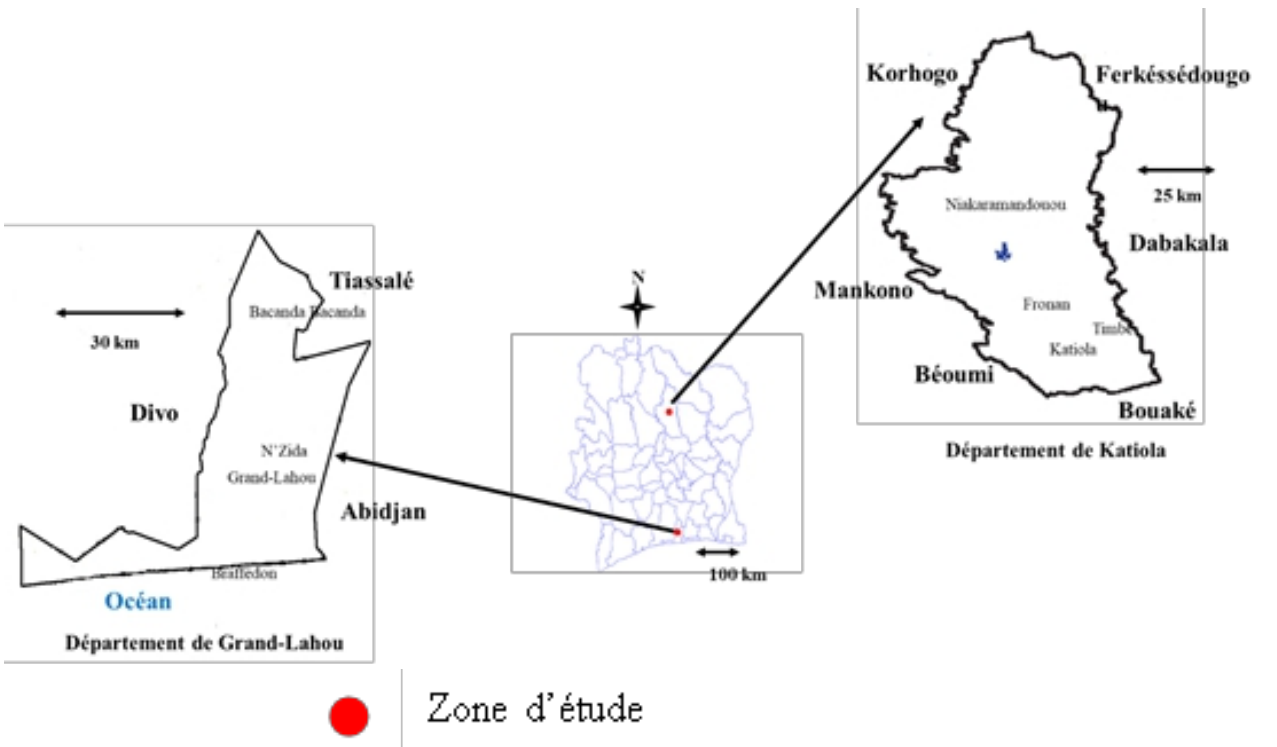

Figure 1 : Situation géographique des départements de Grand-Lahou et de Katiola

\section{Echantillonnage}

L'étude a concernée deux catégories de personnes, les femmes en âge de ménopause et les praticiens de la médecine traditionnelle. Les femmes âgées de 40 à 70 ans ont constitué 1'unité d'échantillonnage. La taille de l'échantillon a été calculée selon la formule suivante :

$\mathbf{n}=\mathbf{t}^{2} \times \mathbf{p}(\mathbf{1}-\mathbf{p}) / \mathbf{m}^{2}$

avec $\mathrm{n}=$ taille d'échantillon requise, $\mathrm{t}=1,96$ à un niveau de confiance de 95 $\%, \mathrm{p}=$ prévalence estimative des femmes en âge de ménopause dans la zone du projet et $\mathrm{m}=$ marge d'erreur à $3,3 \%$.

La prévalence des femmes en âge de ménopause, calculée grâce à la pyramide des âges de la population résidente (INS, 2015) a été de 22,43\%. Ainsi, un échantillon de 267 femmes à enquêter a été obtenu, dont 155 femmes à GrandLahou et 112 femmes à Katiola. Tous les acteurs de la médecine traditionnelle accessibles ont constitué le deuxième échantillon de l'étude. 


\section{Méthode d'enquête ethnobotanique}

Les enquêtes se sont déroulées d'août 2016 à août 2017 à l'aide d'un questionnaire semi-directif. La première partie de l'enquête a concerné les femmes âgées de 40 à 70 ans, non gestantes et non allaitantes vivant sur les différents sites. Elles ont été interrogées sans distinction d'ethnie et de nationalité. Les enquêtées ont été interviewées individuellement afin de garder la confidentialité des informations reçues et d'éviter l'effet d'influence. Le questionnaire a porté sur les données socio-économiques, le niveau de connaissance et la perception de la ménopause, les plantes médicinales ou alimentaires utilisées, les fréquences d'utilisation et le rappel des 24 heures. La réalisation de l'enquête, dans les villages, a été possible grâce à un guide local, qui a servi d'intermédiaire pour faciliter la communication avec les personnes interviewées.

Dans la deuxième partie de l'enquête, des herboristes et des tradipraticiennes ont été interrogés selon leur disponibilité. Le guide d'entretien portait sur la définition de la ménopause, l'âge normal de la ménopause et la manière dont ils reconnaissaient ces symptômes. Les questions ont été posées soit en français ou en langue locale par le biais d'un guide.

\section{Traitement des données}

\section{Niveau de connaissance des symptômes de la ménopause}

Le niveau de connaissance relative des populations $(\mathrm{Cr})$ pour chaque symptôme listé a été calculé par le rapport entre le nombre (n) de personnes connaissant le symptôme et le nombre total $(\mathrm{N})$ de personnes interrogées à travers la formule suivante :

\section{$\mathrm{Cr}=(\mathbf{n} / \mathrm{N}) \times \mathbf{1 0 0}$}

Cette méthode, utilisée par Kouamé et al. (2008), a permis de classer les symptômes connus par les femmes interrogées. Les symptômes avec un niveau de connaissance relative (Cr) compris entre 50 et $100 \%$, sont les plus connus. Ceux avec un $\mathrm{Cr}$ de 25 à $50 \%$, sont dits moyennement connus et pour un $\mathrm{Cr}$ de 0 à $25 \%$, les symptômes sont dits peu connus.

\section{Fréquence de citation}

La fréquence de citation (FC) qui représente le nombre de citations $(\mathrm{N})$ rapporté au nombre total (NT) de répondants a été utilisée pour évaluer l'importance des plantes utilisées et la crédibilité des informations reçues (Smith et al., 1998). Elle a été calculée par la formule suivante :

FC $=$ N/NTx100 


\section{Indice de Smith}

L'indice de Smith a permis d'évaluer l'importance accordée par les répondants à chaque espèce entrant dans les habitudes médicinales des femmes (Malan et al., 2015 ; Sutrop, 2001). C'est un indice qui prend en compte la fréquence de citation, la longueur des listes libres et le rang d'apparition de chaque espèce dans la liste libre (Borgatti, 1996). Il se calcule selon la formule suivante : $\mathbf{S}=\{\boldsymbol{\Sigma}[(\mathbf{L i}-\mathbf{R j}) / \mathbf{L j}]\} / \mathbf{N}$

$\mathrm{S}$ : importance d'une citation ; $\mathrm{Li}$ : longueur d'une liste de citations et $\mathrm{Rj}$ : rang d'une citation dans la liste. L'indice de Smith est calculé à l'aide du logiciel ANTHROPAC version 1.0 (Borgatti, 1996).

\section{Fréquence de consommation}

La fréquence de consommation (FCons) est le rapport entre le nombre de fois (n) que la plante a été consommée par an et le nombre total (nt) de femmes. Elle a été calculée comme suite : $\mathbf{F C o n s}=\mathbf{n} / \mathbf{n t}$. Elle a permis de connaître les plantes alimentaires locales les plus consommées par les femmes.

\section{Analyses statistiques}

La saisie des données a été réalisée avec le logiciel Epidata 3.1 et transférée sur le logiciel SPSS 20.0 pour la mise en place de la base de données. Les analyses statistiques ont été réalisées à l'aide du logiciel X1Stat version 2018.2. La relation qui existe entre le niveau de connaissance des symptômes de la ménopause et des paramètres tels que le statut ménopausique et le niveau d'instruction a été établi en utilisant le test de Khi-deux $\left(\chi^{2}\right)$ au seuil de 0,05 $\%$. Une classification ascendante hiérarchique a été réalisée pour regrouper et classer les plantes en fonction du nombre de symptômes ressentis par les femmes. Elle a permis d'établir une relation entre les plantes et les groupes de femmes formés en fonction du nombre de symptômes ressentis.

\section{Résultats}

\section{Données sociodémographiques}

Les entretiens ont été réalisés auprès de 267 femmes soit 155 à GrandLahou et 112 à Katiola. Ils ont montré, dans 1'ensemble que $126(47,19 \%)$ sont des allochtones, $121(45,32 \%)$ des autochtones et $20(7,49 \%)$ des allogènes. Les enquêtées sont en majorité des ménagères $(78,54 \%)$ contre $16,14 \%$ de commerçantes. La plupart d'entre elles, soit 62,81\%, vivent avec un conjoint et ont plus de six enfants. Un pourcentage de 58,02\% des femmes est analphabète, avec un taux plus élevé à Katiola (62,50 \%). La plupart des femmes 47,96\% ont entre 46 et 55 ans. Sur les 267 femmes interrogées, 88 femmes sont non ménopausées avec un âge variant entre 40 et 55 ans $\pm 3,43$ ans, 12 sont en péri-ménopauses et 167 sont ménopausées. Selon les résultats, l'âge moyen de la ménopause est de 48,88 $\pm 4,19$ ans. Cet âge varie 
significativement en fonction des départements. Il est de, 49,64 $\pm 3,75$ ans à Grand-Lahou, contre 47,71 \pm 4,57 ans à Katiola.

\section{Connaissance de la ménopause par les herboristes}

Au total, neuf herboristes ont été interrogés soit trois à Katiola et six à Grand-Lahou. Elles reçoivent entre 0 et 25 femmes en péri-ménopause ou en ménopause par an. Le diagnostic de la ménopause par ces vendeuses de plantes médicinales est basé principalement sur l'âge de la patiente et les symptômes évoqués par cette dernière. Au total, toutes les vendeuses $(100 \%)$ considèrent que l'absence de règles à partir de 50 ans correspond à la ménopause. Il ressort de ces interviews que ces herboristes assimilent de nombreux symptômes de la ménopause à d'autres maladies telles que le paludisme, les troubles gastrointestinaux qu'elles appellent communément «plaies de ventre », les rhumatismes, les hémorroïdes et les troubles liés au fonctionnement de l'appareil génital de la femme appelé couramment « Bobodouman ». Cela montre que ces dernières ne connaissent pas les symptômes de la ménopause.

\section{Connaissance de la ménopause par les femmes enquêtées}

La majorité des femmes interrogées, soient 96,85\%, estiment que la ménopause est normale à partir de 50 ans. Pour elles, la ménopause est anodine et donc, sans conséquence sur la santé. Elles la considèrent comme une maladie lorsqu'elle apparaît entre 40 et 44 ans (81,42 \%). A Grand-Lahou, 119 femmes (soit 76,77 \%) considèrent la ménopause comme une maladie entre 45 et 49 ans alors qu'à Katiola, 71 femmes $(63,39 \%)$ la considèrent comme normale à cette tranche d'âge. Pour 104 femmes interrogées, soit 90,94 \%, l'arrêt des menstrues est une libération (Tableau 1). La majorité des femmes $(61,00 \%)$ ayant ressentis les symptômes de la ménopause estiment que ces symptômes sont difficilement supportables tandis que 39,00 \% mentionnent le contraire. La distribution des femmes ménopausées et péri-ménopausées selon le nombre de symptômes montre que 40,00 \% ont ressenti entre un et quatre symptômes, près de 32,00 \% ont plus de quatre symptômes et environ 28,00 \% n’ont ressenti aucun symptôme.

Les symptômes et les risques de la ménopause sont méconnus respectivement par 73,68\% et 98,71\% des femmes. Cependant, les femmes de Grand-Lahou (41,94 \%) ont une meilleure connaissance des symptômes de la ménopause comparée à celles de Katiola (10,71\%) (Tableau 1). Le test de Khi-deux réalisé, à cet effet, montre que le niveau de connaissance de la ménopause est fonction du niveau de scolarisation. 
Tableau 1 : Niveau de connaissance des femmes de la ménopause

\begin{tabular}{|c|c|c|c|c|c|}
\hline \multirow{2}{*}{ Paramètres } & & & \multicolumn{2}{|c|}{ Zone d'étude } & \multirow{2}{*}{ Moyennes } \\
\hline & & & Grand-Lahou & Katiola & \\
\hline & & & $\mathrm{N}=155$ & $\mathrm{~N}=112$ & \\
\hline \multirow{6}{*}{$\begin{array}{l}\text { Âge normale } \\
\text { de la } \\
\text { ménopause }\end{array}$} & \multirow{2}{*}[40-44]{} & Oui & 3,23 & 33,93 & 18,58 \\
\hline & & Non & 96,77 & 66,07 & 81,42 \\
\hline & \multirow{2}{*}{ [45 - 49] } & Oui & 23,23 & 63,39 & 43,31 \\
\hline & & Non & 76,77 & 36,61 & 56,69 \\
\hline & \multirow{2}{*}{$50-55[$} & Oui & 95,48 & 98,21 & 96,85 \\
\hline & & Non & 4,52 & 1,79 & 3,15 \\
\hline \multirow{2}{*}{\multicolumn{2}{|c|}{$\begin{array}{l}\text { Connaissance des } \\
\text { symptômes }\end{array}$}} & Oui & 41,94 & 10,71 & 26,32 \\
\hline & & Non & 58,06 & 89,29 & 73,68 \\
\hline \multirow{2}{*}{\multicolumn{2}{|c|}{ Connaissance des risques }} & Oui & 2,58 & 0 & 1,29 \\
\hline & & Non & 97,42 & 100 & 98,71 \\
\hline \multirow{2}{*}{\multicolumn{2}{|c|}{$\begin{array}{c}\text { Sentiment éprouvée à la } \\
\text { ménopause }\end{array}$}} & Libération & 89,03 & 92,86 & 90,94 \\
\hline & & Vieillesse & 10,97 & 7,14 & 9,06 \\
\hline
\end{tabular}

Les femmes les plus instruites (Niveau secondaire) connaissent mieux les symptômes de la ménopause (Figure 2).

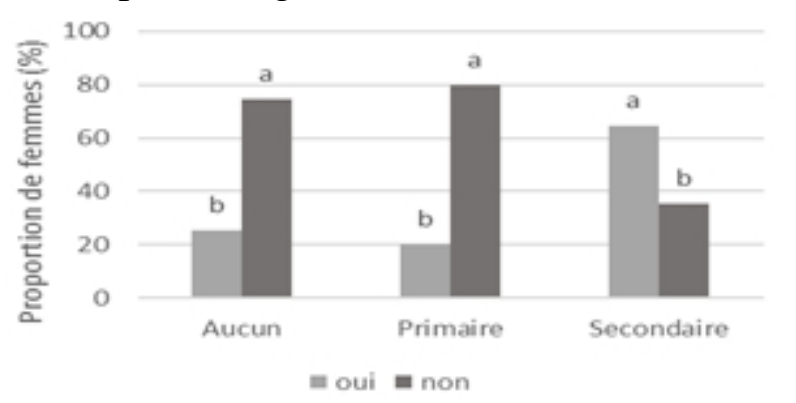

Les bandes portant la même lettre ne montrent pas de différence significative à $\mathrm{P}<$ 0,0001 avec le test de $\mathrm{Khi}^{2}$

Figure 2 : Niveau de connaissance des symptômes de la ménopause en fonction du niveau d'étude

Les résultats montrent que quelques soit leur statut ménopausique et leur niveau d'étude, les femmes ne connaissent pas les risques liés à la ménopause (Figure 3 et 4). Sur un total de 18 symptômes mentionnés par les femmes, les plus cités sont les bouffées de chaleur $(52,00 \%)$ et les douleurs articulaires et musculaires $(28,00 \%)$. Les valeurs du niveau de connaissance relative montrent que les symptômes de la ménopause sont peu connus car elles sont inférieures à $25,00 \%$. 


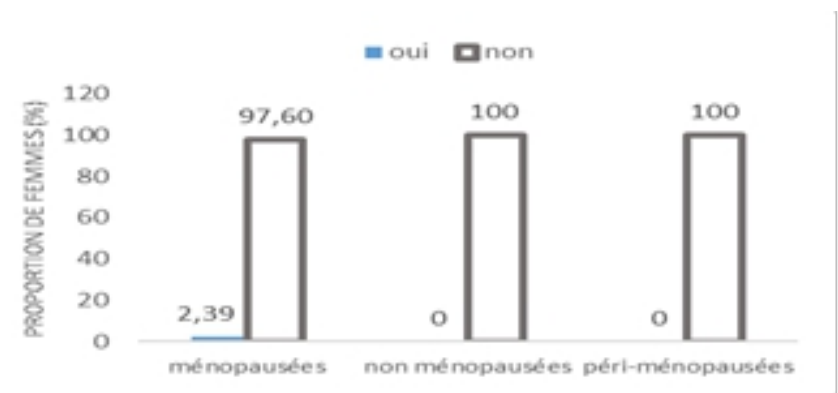

Figure 3 : Niveau de connaissance des risques de la ménopause en fonction du statut ménopausique

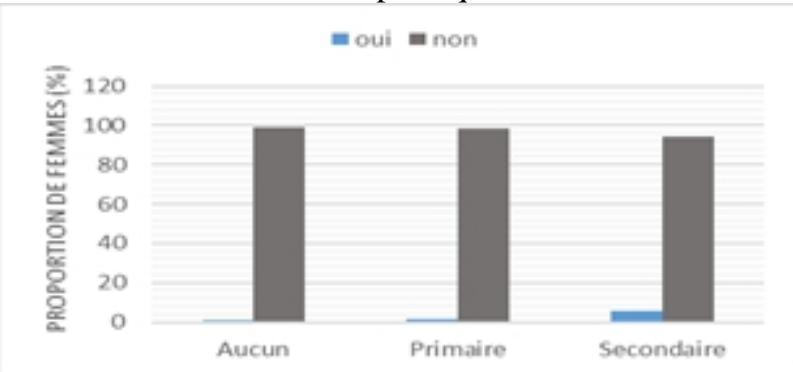

Figure 4 : Niveau de connaissance des risques de la ménopause en fonction du niveau d'étude

Les bouffées de chaleur constituent le symptôme le plus connu avec 28,38 \% de connaissance relative à Grand-Lahou (Tableau 2).

Tableau 2 : Connaissance relative des femmes des symptômes de la ménopause dans les départements de Grand-Lahou et de Katiola

\begin{tabular}{lllll}
\hline \multirow{2}{*}{ No } & \multirow{2}{*}{ Symptômes cités } & \multicolumn{3}{c}{ Niveau de connaissance (\%) } \\
\cline { 3 - 5 } & & Grand-Lahou & Katiola & Moyenne \\
\hline 1 & Bouffées de chaleur & $\mathbf{2 8 , 3 8} \mathbf{1}^{\mathbf{1}}$ & 6,25 & 17,315 \\
2 & Douleurs articulaires et musculaires & 16,12 & 2,67 & 9,395 \\
3 & Fatigues générales & 3,87 & 4,46 & 4,165 \\
4 & Palpitations & 5,8 & 0 & 2,9 \\
5 & Irrégularités menstruelles & 3,87 & 1,78 & 2,825 \\
6 & Céphalées & 3,87 & 0,89 & 2,38 \\
7 & Sueur & 4,51 & 0 & 2,255 \\
8 & Douleurs de ventre & 1,29 & 0,89 & 1,09 \\
9 & Douleurs de bas ventre & 1,93 & 0 & 0,965 \\
10 & Insomnie & 0,64 & 0,89 & 0,765 \\
11 & Ballonnement de ventre & 1,29 & - & 0,645 \\
12 & Nausées & 1,29 & - & 0,645 \\
13 & Absence de règles & 0,64 & - & 0,32 \\
14 & Démangeaisons & 0,64 & - & 0,32 \\
15 & Prise de poids & 0,64 & - & 0,32 \\
16 & Trouble de l'humeur & 0,64 & - & 0,32 \\
17 & Vertiges & 0,64 & - & 0,32 \\
18 & Vomissement & 0,64 & - & 0,32 \\
\hline & 1 Les symptômes avec les niveaux de connaissance en gras sont les plus connus.
\end{tabular}




\section{Diversité des plantes utilisées par les femmes}

Les enquêtes ethnobotaniques réalisées dans les départements de Grand-Lahou et de Katiola ont permis de recenser 113 espèces de plantes médicinales et alimentaires reparties en 95 genres et 44 familles. Les familles les plus représentées sont dans l'ordre d'importance du nombre d'espèces, les Fabaceae (16 espèces), les Solanaceae et les Poaceae (8 espèces) et les Euphorbiaceae (7 espèces). Les 113 espèces végétales sont réparties entre 54 plantes médicinales et 75 plantes alimentaires. Parmi ces espèces, 16 sont à la fois médicinales et alimentaires. Les plantes médicinales inventoriées sont reparties en 52 genres et 24 familles. Les Fabaceae sont les plus représentées avec huit espèces, suivie des Euphorbiaceae avec sept espèces et des Asteraceae avec cinq espèces. Les plantes alimentaires sont réparties en 58 genres et 36 familles. Les Fabaceae et les Solanaceae sont les plus prépondérants avec respectivement neuf et huit espèces. Les groupes d'aliments les plus consommés sont les légumes (28\%), les racines et tubercules $(26 \%)$ et les céréales $(25 \%)$ (Figure 5).

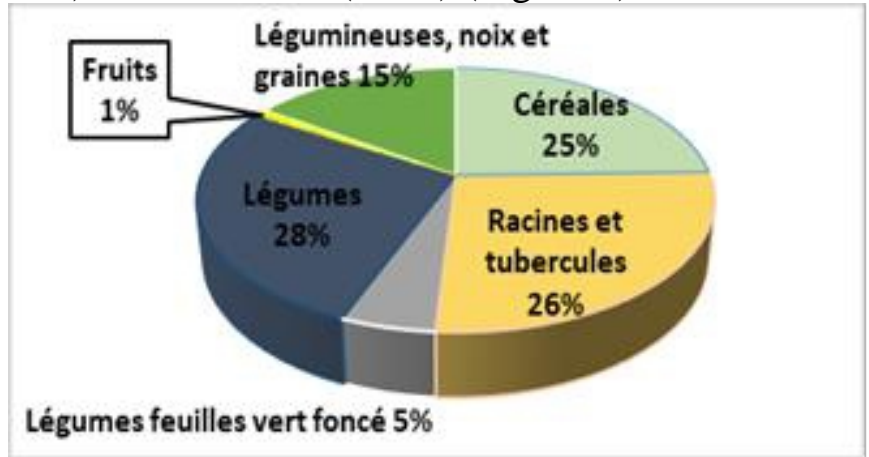

Figure 5 : Spectre des différents groupes d'aliments consommés par les femmes dans les départements de Grand-Lahou et de Katiola

\section{Plantes médicinales utilisées habituellement par les femmes pour leurs soins de santé}

Parmi les 267 femmes interrogées, 187 femmes, dont 130 à GrandLahou et 57 à Katiola, ont mentionné l'utilisation des plantes pour traiter différentes affections dont les symptômes de la ménopause. Ces femmes ont une connaissance moyenne des plantes qu'elles utilisent. Parmi celles qui utilisent des plantes, 138 femmes connaissent le nom des plantes qu'elles utilisent. Les 49 autres $(26,21 \%)$ achètent les plantes sous formes de poudre ou consultent des acteurs de la médecine traditionnelle qui leur donnent soit des décoctés ou des canaris contenant déjà les plantes. Les plantes les plus connues sont Ocimum gratissimum $(\mathrm{S}=0,313$; VU $=3,71)$ et Alchornea cordifolia $(\mathrm{S}=0,154 ; \mathrm{VU}=3,54)$. Ces plantes présentent un indice de Smith et une valeur d'usage élevés (Tableau 3). Les fréquences de citation des espèces utilisées varient de 22,58 \% à $0,64 \%$ à Grand-Lahou et de 6,25 \% à 
$0,89 \%$ à Katiola. Pour leurs soins de santé, les femmes de Grand-Lahou utilisent Ocimum gratissimum $(\mathrm{FC}=67,74 \%)$ et Alchornea cordifolia $(\mathrm{FC}=$ $46,45 \%$ ), qui ont les fréquences de citation les plus élevées. Quant aux femmes de Katiola, elles utilisent majoritairement Azadiratcha indica $(\mathrm{FC}=18,75 \%)$ et Anogeissus leiocarpus ( $\mathrm{FC}=16,07 \%)$, (Tableau 3).

Tableau 3 : Plantes médicinales entrant dans les habitudes des femmes interrogées

\begin{tabular}{|c|c|c|c|c|c|c|c|c|}
\hline \multirow[b]{2}{*}{ Espèces végétales } & \multirow[b]{2}{*}{ Familles } & \multicolumn{3}{|c|}{ Grand-Lahou (GL) } & \multicolumn{3}{|c|}{ Katiola (Kat) } & \multirow{2}{*}{$\begin{array}{l}\text { S } \\
\text { (GL; } \\
\text { Kat) }\end{array}$} \\
\hline & & $\begin{array}{l}\mathrm{FC} \\
(\%) \\
\end{array}$ & VU & $\mathbf{S}$ & \begin{tabular}{|l|}
$\mathrm{FC}$ \\
$(\%)$ \\
\end{tabular} & $\mathbf{V U}$ & $\mathbf{S}$ & \\
\hline Ocimum gratissimum $\mathrm{L}$. & Lamiaceae & 67,74 & 3,71 & 0,371 & 13,39 & 3,4 & 0,152 & $\mathbf{0 , 3 1 3}$ \\
\hline $\begin{array}{l}\text { Alchornea cordifolia } \\
\text { (Schumach. \& Thonn.) } \\
\text { Müll. Arg. }\end{array}$ & Euphorbiaceae & 46,45 & 3,54 & 0,183 & 8,04 & 5 & 0,076 & 0,154 \\
\hline Alstonia boonei De Wild. & Apocynaceae & 32,90 & 4,65 & 0,139 & 0 & 0 & 0 & 0,102 \\
\hline $\begin{array}{l}\text { Vernonia colorata (Willd.) } \\
\text { Dracke }\end{array}$ & Asteraceae & 13,55 & 2,14 & 0,077 & 13,39 & 3,4 & 0,152 & 0,097 \\
\hline Cassia occidentalis L. & Fabaceae & 27,10 & 3,43 & 0,07 & 8,04 & 3,33 & 0,076 & $\mathbf{0 , 0 7 1}$ \\
\hline $\begin{array}{l}\text { Khaya senegalensis (Desr.) } \\
\text { A. Juss. }\end{array}$ & Meliaceae & 13,55 & 4,86 & 0,066 & 2,68 & 4 & 0,03 & 0,056 \\
\hline Moringa oleifera Lam. & Moringaceae & 11,61 & 3,33 & 0,041 & 8,04 & 4,67 & 0,083 & $\mathbf{0 , 0 5 3}$ \\
\hline Tectona grandis $\mathrm{L} . \mathrm{f}$ & Verbenaceae & 25,16 & 2,85 & 0,057 & 2,68 & 3 & 0,015 & 0,046 \\
\hline Heliotropium indicum Linn & Boraginaceae & 11,61 & 2,5 & 0,051 & 2,68 & 3 & 0,015 & $\mathbf{0 , 0 4 1}$ \\
\hline $\begin{array}{l}\text { Anogeissus leiocarpus } \\
\text { (DC.) Guill. Et Perr. }\end{array}$ & Combretaceae & 0 & 0 & 0 & 16,07 & 4,17 & 0,152 & $\mathbf{0 , 0 4}$ \\
\hline $\begin{array}{l}\text { Ricinodendron heudelotii } \\
\text { (Bail.) }\end{array}$ & Euphorbiaceae & 9,68 & 4,8 & 0,055 & 0 & 0 & 0 & $\mathbf{0 , 0 4}$ \\
\hline Azadiratcha indica $\mathrm{L}$. & Meliaceae & 0 & 0 & 0 & 18,75 & 2,86 & 0,136 & $\mathbf{0 , 0 3 6}$ \\
\hline $\begin{array}{l}\text { Bambusa vulgaris Schrad. } \\
\text { ex Wendl. }\end{array}$ & Poaceae & 5,81 & 2 & 0,027 & 2,68 & 3 & 0,03 & $\mathbf{0 , 0 2 8}$ \\
\hline $\begin{array}{l}\text { Cymbopogon citratus (DC.) } \\
\text { Stapf }\end{array}$ & Poaceae & 7,74 & 2,25 & 0,037 & 0 & 0 & 0 & $\mathbf{0 , 0 2 7}$ \\
\hline $\begin{array}{l}\text { Sarcocephalus latifolius } \\
\text { (Sm.) E.A.Bruce }\end{array}$ & Rubiaceae & 3,87 & 3 & 0,022 & 2,68 & 4 & 0,03 & $\mathbf{0 , 0 2 4}$ \\
\hline Musa paradisiaca $\mathrm{L}$. & Musaceae & 3,87 & 1,5 & 0,02 & 2,68 & 1 & 0,03 & $\mathbf{0 , 0 2 3}$ \\
\hline $\begin{array}{l}\text { Chromolaena odorata (L) } \\
\text { King and Robinson }\end{array}$ & Asteraceae & 7,74 & 1,5 & 0,016 & 5,36 & 1,5 & 0,038 & $\mathbf{0 , 0 2 2}$ \\
\hline $\begin{array}{l}\text { Phyllanthus amarus Schum. } \\
\& \text { Thonn. }\end{array}$ & Euphorbiaceae & 15,48 & 4 & 0,027 & 0 & 0 & 0 & $\mathbf{0 , 0 1 9}$ \\
\hline Ageratum conyzoüdes $\mathrm{L}$. & Asteraceae & 0 & 0 & 0 & 5,36 & 3,5 & 0,061 & $\mathbf{0 , 0 1 6}$ \\
\hline $\begin{array}{l}\text { Daniella oliveri (Rolfe) } \\
\text { Hutch. Et Dalziel }\end{array}$ & Fabaceae & 3,87 & 3 & 0,022 & 0 & 0 & 0 & $\mathbf{0 , 0 1 6}$ \\
\hline Mangifera indica $\mathrm{L}$. & Anarcadiaceae & 5,81 & 4,67 & 0,022 & 0 & 0 & 0 & $\mathbf{0 , 0 1 6}$ \\
\hline Persea americana Mill. & Lauraceae & 3,87 & 2 & 0,019 & 0 & 0 & 0 & $\mathbf{0 , 0 1 4}$ \\
\hline Carica papaya $\mathrm{L}$. & Caricaceae & 1,94 & 2 & 0,004 & 8,04 & 2,33 & 0,038 & $\mathbf{0 , 0 1 3}$ \\
\hline Cassia siamea Lam. & Fabaceae & 1,94 & 3 & 0,007 & 2,68 & 2 & 0,03 & $\mathbf{0 , 0 1 3}$ \\
\hline Lippia multiflora Moldenke & Verbenaceae & 3,87 & 2 & 0,018 & 0 & 0 & 0 & 0,013 \\
\hline Cajanus cajan (L.) Millsp & Fabaceae & 5,81 & 3,33 & 0,012 & 2,68 & 3 & 0,01 & 0,012 \\
\hline
\end{tabular}




\begin{tabular}{|c|c|c|c|c|c|c|c|c|}
\hline $\begin{array}{l}\text { Kalanchoe crenata } \\
\text { (Andrews) Haw. var. } \\
\text { crenata }\end{array}$ & Crassulaceae & 3,87 & 1,5 & 0,016 & 0 & 0 & 0 & $\mathbf{0 , 0 1 2}$ \\
\hline Spondias mombin L. & Anacardiaceae & 5,81 & 3 & 0,016 & 0 & 0 & 0 & 0,012 \\
\hline $\begin{array}{l}\text { Parkia biglobosa (Jacq.) R. } \\
\text { Br. Ex G. Don }\end{array}$ & Fabaceae & 1,94 & 3 & 0,004 & 2,68 & 5 & 0,023 & 0,009 \\
\hline Adansonia digitata $\mathrm{L}$. & Bombacaceae & 0 & 0 & 0 & 2,68 & 4 & 0,03 & $\mathbf{0 , 0 0 8}$ \\
\hline Aloes verra L. Bum f. & Xanthorrhceae & 0 & 0 & 0 & 2,68 & 5 & 0,03 & 0,008 \\
\hline Bidens pilosa $\mathrm{L}$. & Asteraceae & 1,94 & 2 & 0,011 & 0 & 0 & 0 & 0,008 \\
\hline Bridelia ferruginea Benth. & Euphorbiaceae & 1,94 & 5 & 0,011 & 0 & 0 & 0 & 0,008 \\
\hline Elaeis guineensis (Jacq) & Arecaceae & 1,94 & 5 & 0,011 & 0 & 0 & 0 & $\mathbf{0 , 0 0 8}$ \\
\hline Morinda lucida Benth & Rubiaceae & 1,94 & 5 & 0,011 & 0 & 0 & 0 & 0,008 \\
\hline $\begin{array}{l}\text { Parquetina nigrescens } \\
\text { (Afzel.) Bullock }\end{array}$ & Periplocaceae & 5,81 & 4 & 0,011 & 0 & 0 & 0 & 0,008 \\
\hline $\begin{array}{l}\text { Piliostigma thonninguii } \\
\text { (Schumach.) Milne-Redh. }\end{array}$ & Fabaceae & 1,94 & 4 & 0,011 & 0 & 0 & 0 & $\mathbf{0 , 0 0 8}$ \\
\hline $\begin{array}{l}\text { Piptadeniastrum africanum } \\
\text { (Hook.f.) Brenan }\end{array}$ & Fabaceae & 1,94 & 5 & 0,011 & 0 & 0 & 0 & $\mathbf{0 , 0 0 8}$ \\
\hline $\begin{array}{l}\text { Pseudathria hookeri Wight } \\
\text { \& Arn. }\end{array}$ & Fabaceae & 1,94 & 2 & 0,011 & 0 & 0 & 0 & $\mathbf{0 , 0 0 8}$ \\
\hline Annona muricata $\mathrm{L}$. & Annonaceae & 1,94 & 2 & 0,009 & 0 & 0 & 0 & 0,006 \\
\hline $\begin{array}{l}\text { Hallea stipulosa (DC.) } \\
\text { Leroy }\end{array}$ & Rubiaceae & 1,94 & 4 & 0,009 & 0 & 0 & 0 & 0,006 \\
\hline $\begin{array}{l}\text { Landolphia owariensis } \mathrm{P} . \\
\text { Beauv. }\end{array}$ & Apocynaceae & 3,87 & 4,5 & 0,008 & 0 & 0 & 0 & 0,006 \\
\hline Ricinus communis L. & Euphorbiaceae & 3,87 & 3,5 & 0,055 & 0 & 0 & 0 & 0,006 \\
\hline Annona senegalensis Pers. & Annonaceae & 0 & 0 & 0 & 2,68 & 5 & 0,02 & 0,005 \\
\hline Jatropha curcas L. & Euphorbiacaea & 1,94 & 2 & 0,007 & 0 & 0 & 0 & 0,005 \\
\hline Lannea acida A. Rich. & Anacardiaceae & 3,87 & 5 & 0,007 & 0 & 0 & 0 & 0,005 \\
\hline $\begin{array}{l}\text { Lantana camara L. var. } \\
\text { camara }\end{array}$ & Verbenaceae & 1,94 & 5 & 0,005 & 0 & 0 & 0 & 0,004 \\
\hline Manihot esculenta Crantz & Euphorbiaceae & 1,94 & 1 & 0,005 & 0 & 0 & 0 & 0,004 \\
\hline Rovolfia vomitoria Afzel. & Apocynaceae & 1,94 & 3 & 0,005 & 0 & 0 & 0 & 0,004 \\
\hline $\begin{array}{l}\text { Thevetia neriifolia Juss. ex } \\
\text { Steud. }\end{array}$ & Apocynaceae & 1,94 & 2 & 0,005 & 0 & 0 & 0 & 0,004 \\
\hline $\begin{array}{l}\text { Tithonia diversifolia } \mathrm{A} . \\
\text { Gray }\end{array}$ & Asteraceae & 1,94 & 2 & 0,005 & 0 & 0 & 0 & 0,004 \\
\hline $\begin{array}{l}\text { Alternanthera pengens } \\
\text { Kunth }\end{array}$ & Amaranthaceae & 1,94 & 5 & 0,004 & 0 & 0 & 0 & 0,003 \\
\hline Amaranthus spinosus L. & Amaranthaceae & 1,94 & 2 & 0,004 & 0 & 0 & 0 & 0,003 \\
\hline Passiflora edulis Sims & Passifloraceae & 1,94 & 3 & 0,002 & 0 & 0 & 0 & 0,002 \\
\hline
\end{tabular}

FC : Fréquence de citation; VU : Valeur d'usage ; S : Indice de Smith

Le dendrogramme réalisé pour classer les plantes en fonction du nombre de symptômes ressentis par les femmes montre que les espèces végétales sont reparties en quatre groupes. Les groupes I et II sont composés des espèces majoritairement utilisées par les femmes ayant entre 1 et 4 symptômes. Ils sont représentés, au total, par 18 espèces (34 \%). Les plus homogènes sont, Khaya senegalensis, Tectona grandis et Spondias mombin 
pour le groupe I ; et Ricinodendron heudelotii, Ricinus communis et Tithonia diversifolia pour le groupe II. Les espèces végétales du groupe III sont les plus utilisées par les femmes n'ayant ressenti aucun symptôme. Ce groupe représente $56 \%$ de l'ensemble des espèces médicinales utilisées par les femmes interrogées. Cela montre que les plantes médicinales entrent dans les habitudes des femmes n'ayant pas de symptômes pendant la ménopause. Le groupes IV est constitué des espèces les plus utilisées par les femmes ayant plus de quatre symptômes. Il renferme Bidens pilosa, Lantana camara, Lippia multiflora, Bridelia ferruginea et Parquetina nigrescens (Figure 6).

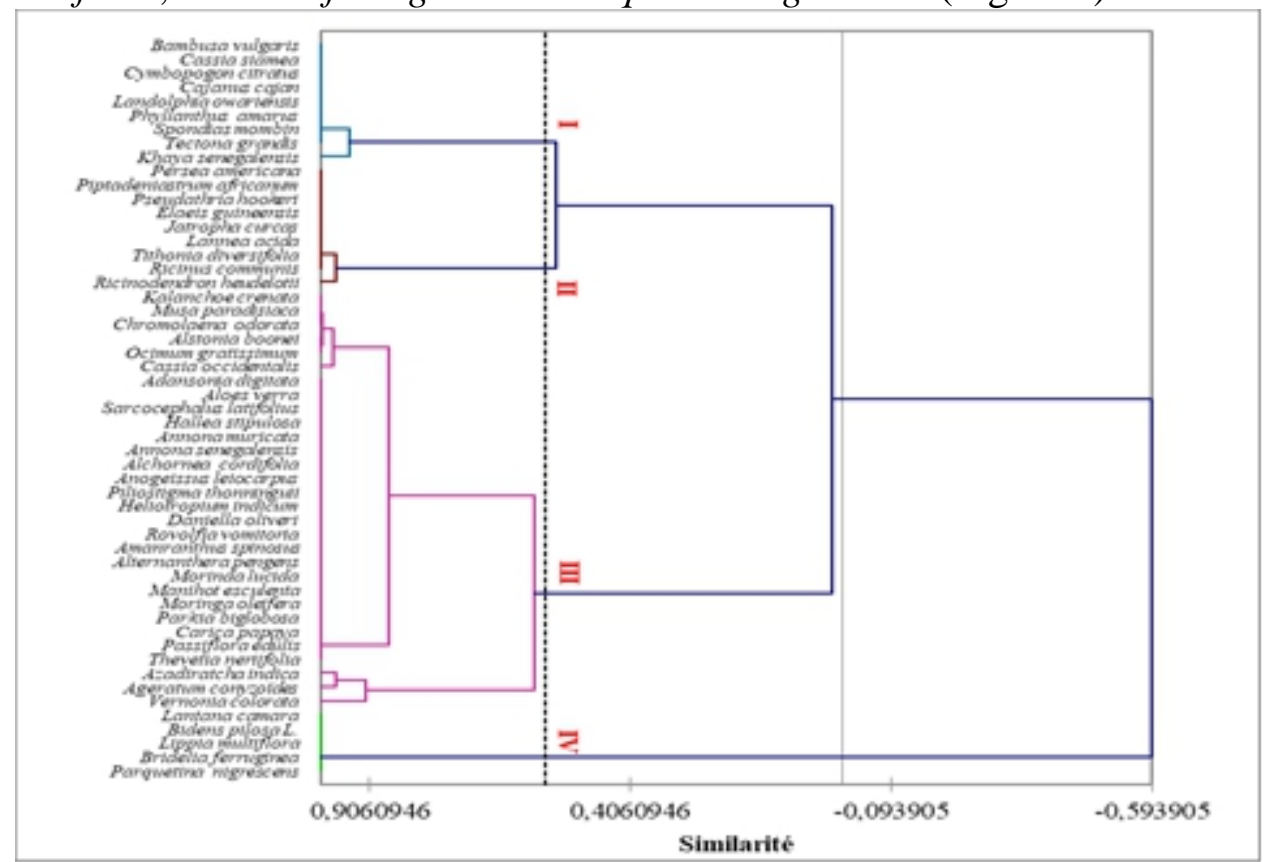

Figure 6 : Dendrogramme des différentes classes de plantes médicinales en fonction du nombre de symptômes ressentis par les femmes ménopausées

L'analyse du dendrogramme montre que de nombreuses plantes entrent dans les habitudes médicinales des femmes n'ayant pas de symptômes par rapport à celles qui en ont eu plus de quatre. L'utilisation de plusieurs plantes pourraient avoir un effet dans la réduction des symptômes de la ménopause. La distribution des familles botaniques en fonction des classes de femmes ménopausées montre que les familles des Fabaceae, des Apocynaceae et des Asteraceae sont majoritairement utilisées par les femmes n'ayant ressenti aucun symptôme (Figure 7). 


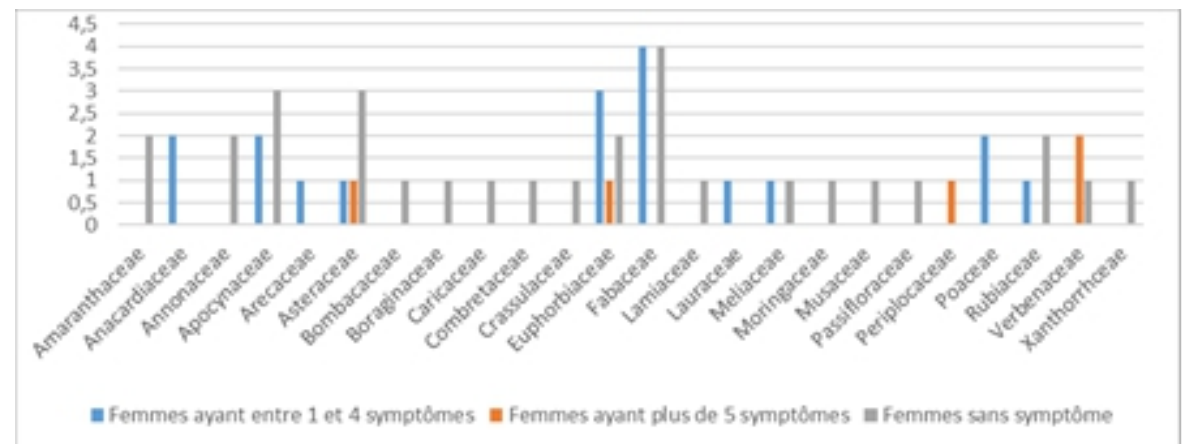

Figure 7 : Distribution des familles botaniques en fonction du nombre de symptômes ressentis par les femmes ménopausées

Dans les deux localités, les femmes utilisent ces espèces pour traiter plusieurs affections dont les plus citées sont le paludisme et les plaies de ventre (troubles gastro-intestinaux) (Figure 8). De cet histogramme, il ressort que des symptômes de la ménopause, les plus traités, sont les douleurs articulaires et musculaires, la fatigue générale, les bouffées de chaleur. Les organes de plantes les plus utilisés dans les préparations sont les feuilles $(73,24 \%)$ et les écorces de tiges $(20,42 \%)$. La décoction (59\%) et le broyage (31\%) sont les modes de préparation les plus utilisées. Les femmes administrent majoritairement les recettes de plantes par voie orale (52\%) avec une prise moyenne de deux fois par jour; ou par voie rectale $(38 \%)$ avec une prise moyenne de deux fois par semaine. Les traitements durent, en moyenne, une semaine.

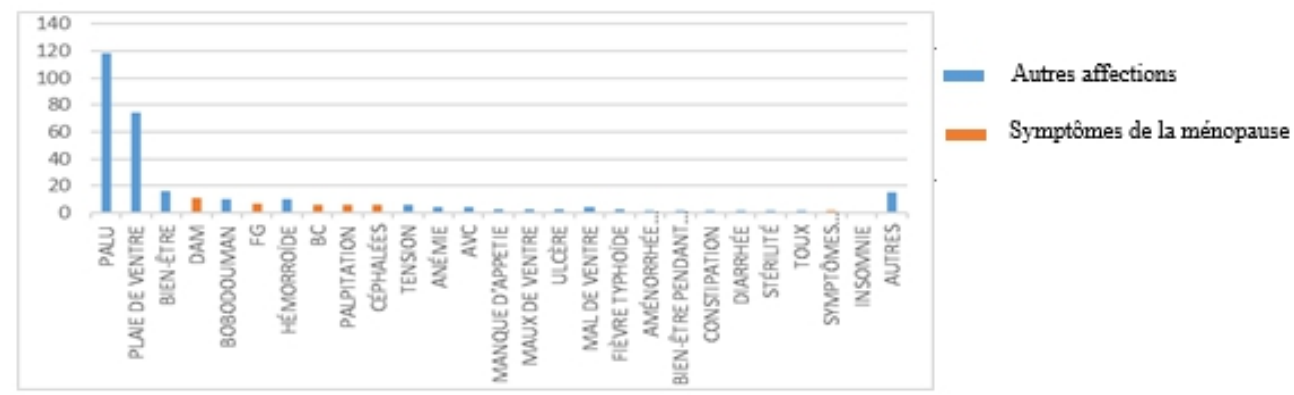

Figure 8 : Affections traitées par les femmes

\section{Régime alimentaire des femmes}

Les femmes de Grand-Lahou ont une alimentation basée sur le manioc (Manihot esculenta) $(88,39 \%)$ consommé généralement sous forme « d'attiéké ». L'attiéke est obtenu à partir de la pâte fermentée de tubercule de manioc. Celles de Katiola ont une alimentation basée sur le maïs (Zea mays) $(96,43 \%)$ consommé généralement sous forme de «tô ». Cet aliment est issu de la poudre des grains de maïs cuits à l'eau. 
Les principales sauces consommées par les femmes interrogées sont composées pour la plupart de légumes tels que l'aubergine (Solanum spp, $93.03 \%$ ) et le gombo (Abelmoschus esculentus) (91,59\%). La sauce graine préparée à partir de la pulpe de noix de palme (Elaeis guineensis) $(86,11 \%$ ) et la sauce arachide (Arachis hypogea) $(84,91 \%)$ arrivent en seconde position.

Les fruits les plus consommées sont la mangue (Mangifera indica) (89,60\%), l'orange (Citrus sinensis) (70,57\%), l'avocat (Persea americana) $(64,79 \%)$ et la banane douce (Musa paradisiaca) $(52,81 \%)$.

Sur les 75 espèces alimentaires inventoriées, une liste de 29 plantes locales $(38,66 \%)$ couramment consommées par les femmes ménopausées a été dressée. Ces plantes sont réparties en 26 genres et 21 familles. La famille des Fabaceae et des Solanaceae sont les plus représentées avec trois espèces chacune.

Les fréquences de citation de ces plantes varient entre $83,67 \%$ et 1,36 $\%$. Parmi les 10 plantes les plus citées dans les deux localités, Elaeis guineensis $(\mathrm{FC}=83,67 \%)$ et Solanum anomalum $(\mathrm{FC}=62,02 \%)$ ont les fréquences de citation les plus élevées. Ces plantes sont à la fois disponibles dans les deux départements. A Grand-Lahou, ce sont, Elaeis guineensis (FC= $89,09 \%)$, Ricinodendron heudelotii $(\mathrm{FC}=45,45 \%)$ et Ipomoea batatas $(42,73$ $\%)$ qui ont les fréquences de citation les plus élevées. Leurs fréquences de consommation sont, respectivement, 79,63;85,70 et 23,48. Par contre à Katiola, ce sont Corchorus olitorius $(\mathrm{FC}=86,95 \%$; Fcons $=49,86)$, Solanum nigrum $(\mathrm{FC}=85,50 \%$; Fcons $=75,81)$ et Parkia biglobosa $(\mathrm{FC}=85,50 \%$, Fcons $=132,1)$ qui ont obtenu les fréquences de citation les plus élevées (Tableau 4).

Tableau 4 : Plantes alimentaires consommées par les femmes ménopausées

\begin{tabular}{|c|c|c|c|c|c|c|c|}
\hline \multirow{3}{*}{ Espèces végétales } & \multirow{3}{*}{ Familles } & \multicolumn{6}{|c|}{ Zone d'étude } \\
\hline & & \multicolumn{2}{|c|}{ Grand-Lahou } & \multicolumn{3}{|c|}{ Katiola } & \multirow[b]{2}{*}{$\begin{array}{c}\text { Disponibilité } \\
\text { (Statut) }\end{array}$} \\
\hline & & $\begin{array}{l}\text { FC } \\
(\%) \\
\end{array}$ & Fcons/an & $\begin{array}{c}\text { Disponibilité } \\
\text { (Statut) }\end{array}$ & $\begin{array}{l}\text { FC } \\
(\%) \\
\end{array}$ & Fcons/an & \\
\hline Parkia biglobosa (Jacq.) G.Don & Fabaceae & 17,25 & 11,41 & $\mathrm{P}$ & 88,41 & 132,1 & $\mathrm{D}$ \\
\hline Elaeis guineensis (Jacq) & Arecaceae & 89,09 & 79,63 & $\mathrm{D}$ & 78,26 & 30,99 & $\mathrm{D}$ \\
\hline Solanum anomalum Thonn. & Solanaceae & 40 & 45,55 & $\mathrm{D}$ & 84,06 & 61,13 & $\mathrm{D}$ \\
\hline $\begin{array}{l}\text { Ricinodendron heudelotii (Baill.) } \\
\text { Heckel }\end{array}$ & Euphorbiaceae & 45,45 & 85,70 & $\mathrm{D}$ & 40,58 & 20,38 & $\mathrm{P}$ \\
\hline Hibiscus sabdariffa L. & Malvaceae & 31,82 & 21,17 & $\mathrm{D}$ & 84,06 & 68,36 & $\mathrm{D}$ \\
\hline Solanum nigrum L. & Solanaceae & 19,09 & 3,79 & $\mathrm{P}$ & 85,51 & 75,81 & $\mathrm{D}$ \\
\hline Corchorus olitorius L. & Tiliaceae & 35,45 & 25,66 & $\mathrm{P}$ & 86,96 & 49,86 & $\mathrm{D}$ \\
\hline Amaranthus hybridus L. & Amaranthaceae & 20,91 & 13,84 & $\mathrm{D}$ & 59,42 & 22,78 & $\mathrm{D}$ \\
\hline Ipomoea batatas $\mathrm{L}$ & Convolvulaceae & 42,73 & 23,48 & $\mathrm{D}$ & 33,33 & 6,45 & $\mathrm{P}$ \\
\hline Colocasia esculenta (L.) Schott. & Araceae & 25,45 & 10,00 & $\mathrm{P}$ & 39,13 & 14,57 & $\mathrm{P}$ \\
\hline Ceiba pentandra (L.) Gaertn. & Bombacaceae & 6,36 & 1,25 & $\mathrm{P}$ & 73,91 & 22,67 & $\mathrm{D}$ \\
\hline Solanum torvum $\mathrm{Sw}$ & Solanaceae & 20 & 19,35 & $\mathrm{P}$ & 0 & 0 & nd \\
\hline Basella alba L. & Basellaceae & 16,36 & 8,63 & $\mathrm{P}$ & 15,94 & 2,64 & $\mathrm{P}$ \\
\hline Cucurbita maxima Duchesne & Cucurbitaceae & 0,91 & 0,02 & $\mathrm{P}$ & 34,78 & 8,42 & $\mathrm{D}$ \\
\hline Borassus aethiopum Mart. & Poaceae & 9,09 & 0,50 & $\mathrm{D}$ & 50,72 & 4,62 & $\mathrm{D}$ \\
\hline Pistacia vera $\mathrm{L}$. & Anacardiaceae & 2,73 & 0,18 & $\mathrm{P}$ & 40,58 & 3,07 & $\mathrm{P}$ \\
\hline
\end{tabular}


Manihot esculenta Crantz

Adansonia digitata $\mathrm{L}$.

Cleome gynandra $\mathrm{L}$.

Talinum fruticosum (L.) Juss.

Pisum sativum L.

Vernonia colorata (Willd.)

Dracke

Annona muricata $\mathrm{L}$.

Ficus exasperata Vahl

Irvingia gabonensis (Aubry

Leconte ex O'Rorke) Baill.

Abelmoschus esculentus $\mathrm{L}$.

Landolphia hirsuta (Hua)

Pichon

Annona senegalensis Pers.

Spondias monbin L.

\begin{tabular}{lccc|ccc} 
Euphorbiaceae & 14,55 & 3,19 & $\mathrm{D}_{1}$ & 0 & 0 & $\mathrm{nd}$ \\
Bombacaceae & 4,55 & 0,14 & $\mathrm{P}$ & 26,09 & 2,91 & $\mathrm{D}$ \\
Cleomaceae & 0 & 0 & $\mathrm{nd}$ & 24,64 & 2,88 & $\mathrm{P}$ \\
Talinaceae & 17,27 & 2,80 & $\mathrm{D}_{1}$ & 0 & 0 & $\mathrm{nd}$ \\
Fabaceae & 3,64 & 0,07 & $\mathrm{P}$ & 47,83 & 2,46 & $\mathrm{D}$ \\
Asteraceae & 3,64 & 1,66 & $\mathrm{D}$ & 0 & 0 & $\mathrm{nd}$ \\
& 11,82 & 0,40 & $\mathrm{D}$ & 28,99 & 0,94 & $\mathrm{D}$ \\
Annonaceae & 0,91 & 0,01 & $\mathrm{P} 1$ & 7,25 & 1,13 & $\mathrm{P}_{1}$ \\
Moraceae & 10,91 & 1,10 & $\mathrm{P}$ & 1,45 & 0,03 & $\mathrm{P}$ \\
Irvingiaceae & & & & & & \\
Malvaceae & 2,73 & 0,28 & $\mathrm{D}_{1}$ & 0 & 0 & $\mathrm{nd}$ \\
Apocynaceae & 0,91 & 0,02 & $\mathrm{D}$ & 4,35 & 0,19 & $\mathrm{D}$ \\
& 4,55 & 0,14 & $\mathrm{P} 1$ & 5,80 & 0,04 & $\mathrm{P}_{1}$ \\
Annonaceae & 0 & 0 & $\mathrm{nd}$ & 2,90 & 0,06 & $\mathrm{P}_{1}$ \\
Anacardiaceae & 0 & &
\end{tabular}

FC : Fréquence de citation ; FCons : Fréquence de consommation, D : Disponible, P : Peu disponible ; 1 : Dans les champs ; nd : non déterminé

Le dendrogramme réalisé pour classer les plantes alimentaires locales en fonction du nombre de symptômes ressentis par les femmes montre que les espèces végétales sont reparties en trois groupes. Les plantes du groupe I sont majoritairement consommées par les femmes qui ont eu plus de quatre symptômes. Ce sont entre autres Adansonia digitata, Landolphia hirsuta et Annona muricata. Le groupe II représente les espèces végétales les plus consommées par les femmes ayant ressenti entre 1 et 4 symptômes. Il est représenté par certaines espèces telles que Vernonia colorata, Amaranthus hybridus et Annona senegalensis. Le groupe III est constitué des espèces les plus consommées par les femmes qui n'ont présenté aucun symptôme. Il est constitué entre autres de Ricinodendron heudelotii, Abelmoschus esculentus et Basella alba (Figure 9).

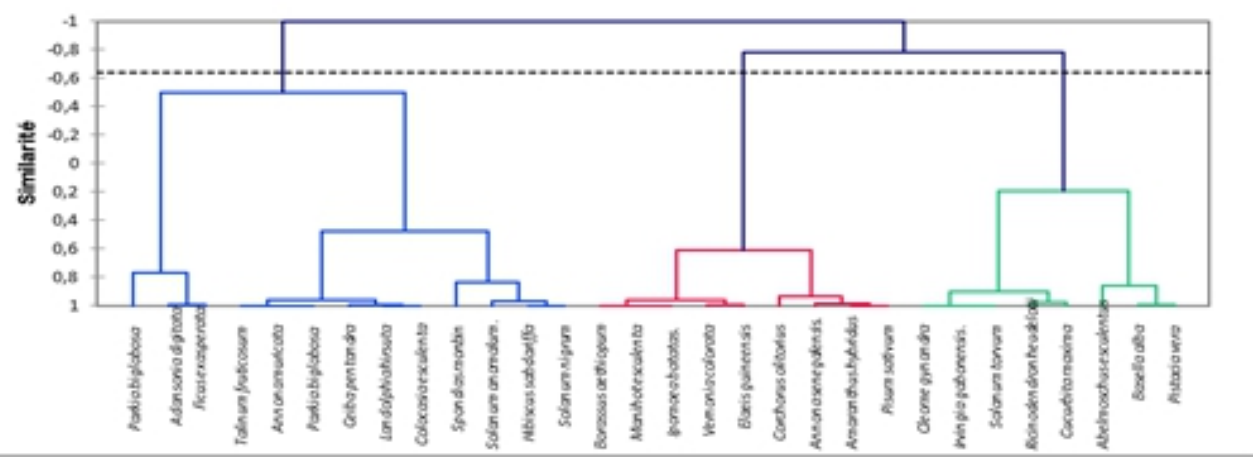

Figure 9 : Dendrogramme des différentes classes de plantes alimentaires en fonction du nombre de symptômes ressenti par les femmes ménopausées 


\section{Discussion}

L'objectif de ce travail était de déterminer le niveau de connaissance des femmes sur la ménopause et les plantes alimentaires et médicinales locales entrant dans les habitudes des femmes en âge de ménopause.

Les enquêtes ont révélé que l'âge moyen de la ménopause dans les deux départements est de 48,88 ans. Cet âge est pratiquement identique à la moyenne internationale qui est 49 ans (Thomas et al., 2001). Sur les 267 femmes enquêtées, 167 étaient ménopausées. La plupart des femmes (96,85 $\%$ ) estiment que la ménopause est normale à partir de 50 ans. La perception des femmes par rapport à l'âge normal de la ménopause pourrait être en rapport avec leur vécu et les informations relayées par leur communauté (Massé, 1995). Cependant, pour ces femmes, la ménopause, lorsqu'elle s'accompagne de signes, autres que les bouffées de chaleur, est anormale quelques soit l'âge. Cette conception pourrait se justifier par une méconnaissance liée à l'insuffisance d'information sur la ménopause et particulièrement sur les symptômes qui l'accompagnent. Si dans certains pays africains comme le Maroc, l'Algérie (Zohra, 2007), des sensibilisations en rapport avec la ménopause sont menées, cela n'est pas encore le cas en Côte d'Ivoire (Pouamon, 2017).

Cette étude a montré que 90,94\% des femmes interrogées perçoivent l'arrêt des menstrues comme une libération. Cette perception des femmes ivoiriennes est identique à celle des algériennes (66,6 \%) (Zohra, 2007), suisses et camerounaises (100\%) (Mbarga 2014).

Pour $61,00 \%$ des femmes enquêtées, les symptômes du climatère sont difficilement supportables contre 39,00\% qui ont un avis contraire. Cette divergence pourrait se justifier par le fait que la ménopause est vécue différemment par chaque femme (OMS, 1996 ; Zohra, 2007, Mbarga 2014) et que les symptômes ressentis varient d'une femme à l'autre (Loukid et al., 2007). En plus, certaines conditions de vie telles qu'une bonne alimentation, le niveau social, la pratique du sport favorisent le fait que certaines femmes supportent les effets de la ménopause et d'autres non (Zohra, 2007).

Ce travail a aussi montré que les femmes, de manière générale, ont peu de connaissance sur les symptômes et les risques liés au retour d'âge. Ce faible niveau de connaissance pourrait être dû au fait que ces dernières ne lient pas forcément les symptômes qu'elles ressentent à ce nouvel état physiologique. Par ailleurs, le caractère tabou de la ménopause dans beaucoup de communautés africaines comme celles de la Côte d'Ivoire (Pouamon, 2017), serait un autre facteur qui justifierait ce niveau de connaissance bas.

Les femmes instruites ont une meilleure connaissance de ces symptômes par rapport aux femmes non instruites. Sanou et al. (2015), mentionnent que l'instruction améliore les chances d'avoir une meilleure culture sanitaire. Les herboristes assimilent les symptômes de la ménopause à 
d'autres maladies telles que le paludisme, les plaies de ventre (troubles gastrointestinaux), les rhumatismes, les hémorroïdes et des troubles liés au fonctionnement de l'appareil génital de la femme. Cette confusion est en rapport avec la similarité entre les symptômes de la ménopause et ceux de certaines maladies.

Au total, 54 plantes médicinales entrant dans les habitudes des femmes ont été recensées. Ces espèces sont reparties en 52 genres et 24 familles. La famille des Fabaceae et des Euphorbiaceae sont les plus représentées. Ces familles font parties, du point de vue spécifique, des plus importantes de la flore ivoirienne (Aké- Assi, 2001). Selon N'Guessan et al., (2010), elles comptent le plus grand nombre de plantes médicinales, traditionnellement utilisées pour leurs effets ocytociques. Parmi les 54 espèces qui entrent dans les soins de santé des femmes, Ocimum gratissimum $(\mathrm{S}=0,313)$ et Alchornea cordifolia $(\mathrm{S}=0,154)$ sont les plus utilisées par les femmes. Les travaux de Lagou et al. (2016) montrent que ces deux plantes sont les plus utilisées dans le traitement des fistules obstétricales dans le district d'Abidjan. Ceci est certainement lié à leur propriété thérapeutique, mais aussi à leur disponibilité dans les formations végétales environnantes. Aussi, Ocimum gratissimum est une plante cultivée.

L'analyse du dendrogramme des plantes médicinales a permis d'établir quatre groupes de plantes. Les espèces végétales du groupe III sont les plus utilisées par les femmes n'ayant eu aucun symptôme. Ce groupe représente 56 $\%$ de l'ensemble des espèces médicinales utilisées par les femmes interrogées. Cela traduit le fait que l'utilisation habituelle des plantes médicinales pourraient avoir un effet dans la réduction des symptômes de la ménopause. L'analyse du dendrogramme des plantes alimentaires a permis de les classer en trois groupes. Les plantes du groupe I et II qui sont respectivement consommées par les femmes n'ayant connu aucun symptôme et celles ayant ressenti entre 1 et 4 symptômes, pourraient jouer un rôle dans le contrôle des symptômes de la ménopause. Les plantes médicinales et alimentaires renferment de nombreux principes actifs qui ont des activités thérapeutiques complémentaires ou synergiques capables de guérir ou de prévenir certaines maladies. La distribution des familles botaniques en fonction des classes de femmes ménopausées montre que les familles des Fabaceae, des Apocynaceae et des Asteraceae sont majoritairement utilisées par les femmes n'ayant aucun symptôme. Ces familles de plantes sont caractéristiques des flavonoïdes (Kidik et al., 2015) et plus spécifiquement des isoflavonoïdes (Lapcik et al., 2007). La présence de ces composées chimiques dans les espèces de plantes appartenant à ces familles serait bénéfique dans la réduction ou l'absence des symptômes de la ménopause. Ces composés sont connus comme des phytoestrogènes qui améliorent l'état des femmes à la ménopause. Les flavonoïdes, par leur activité antioxydante prononcée (Yue et al., 2010), sont 
capables de lutter contre certains symptômes de la ménopause tels que le vieillissement cutané (Sacks et al., 2006). Ces composés, notamment les isoflavones sont également capables d'améliorer les troubles vasomoteurs en diminuant l'intensité et la fréquence des bouffées de chaleur (Amiot et al., 2012), de réduire la résorption osseuse et de stimuler la formation osseuse (Lecerf, 2007). Les polyphénols, de par leurs activités anti-inflammatoires et analgésiques (Babar Ali et al., 2007), sont capables de soulager les femmes des douleurs musculaires et articulaires qu'elles pourraient ressentir.

\section{Conclusion}

Le présent travail a permis de connaître le niveau de connaissance des femmes de la ménopause et les plantes médicinales et alimentaires locales entrant dans les habitudes des femmes ménopausées. Pour les femmes enquêtées, la ménopause doit être anodine et commencer vers 50 ans. Les symptômes et les risques liés à la ménopause sont, en général, méconnus des femmes. La ménopause qui s'accompagne de signes autre que les bouffées de chaleur sont anormales quelques soit l'âge. Ces bouffées de chaleur représentent le symptôme le plus connu des femmes. Le niveau d'instruction joue un rôle dans la bonne connaissance des différents symptômes. Les femmes perçoivent la ménopause comme une libération si elle ne s'accompagnait pas de signes, qui sont le plus souvent difficiles à supporter. Concernant les habitudes médicinales et alimentaires des femmes, il en ressort que 47 plantes sont majoritairement utilisées par les femmes ménopausées n'ayant pas présenté de symptômes ou présentant très peu de symptômes de la ménopause. Ces plantes pourraient avoir un effet sur les symptômes de la ménopause. La recherche de composés phytoestrogéniques dans ces plantes permettra de mettre en relation l'utilisation de ces plantes et la réduction des symptômes de la ménopause.

\section{Remerciements}

Les auteurs tiennent à remercier 1'Association de Recherche OuestAfricaine (WARA) pour son appui financier.

\section{References:}

1. Aké-Assi L. 2001. Flore de la Côte-d'Ivoire : catalogue systématique, biogéographique et écologique. Boissiera, Conservatoire et Jardin Botanique de Genève, 396 p.

2. Al-Eassa Abeer A., Al-Fadel Abeer M., Al-Ajmi Maryam A., AlNajjar Anwar A., Makboul Gamal M., Elshazly Medhat. 2012. Connaissance et attitude des médecins de premier recours en matière de prise en charge des symptômes postménopausiques. Alexandria Medical Journal. 48(2): 167-73. 
3. Amiot M.-J., Coxam V., Strigler F. 2012. Les phytomicronutriments. Edition Lavoisier, 408 p.

4. Babar Ali M., Hahn E.J., Paek K.Y. 2007. Methyl Jasmonate and Salicylic Acid Induced Oxidative Stress and Accumulation of Phenolics in Panax ginseng Bioreactor Root Suspension Cultures. Molecules. 12: 607-621.

5. Borgatti P.S. 1996. Anthropac 4.0. Natick, M.A: Analytic Technologies. Elicitation technique for cultural domain analysis. In the ethnographer's toolkit, J. SCHENSUL, M. LE COMPTE, S. BORGATTI and B. NASTASI (Eds), 3: 115 - 151.

6. Bumbu A., Paşca B., Ţiţ D.M., Bungău S., Bumbu G. 2016. Les effets des isoflavones de soja et du traitement hormonal substitutif sur l'incidence et l'évolution de l'incontinence urinaire féminine postménopausique. Farmacia. 64(3): 419-22.

7. Delia M.I., Annamaria P., Ciprian I., Gheorghe F., Cristian F., Simona B. 2017. Evolution des symptômes somatiques et végétatifs chez les femmes ménopausées traitées par phytoestrogènes et thérapie hormonale substitutive. Irania Journal Santé publique. 46(11): 15281534.

8. Drapier-Faure E. 2003. La ménopause. Masson, 2ème édition, 320 p.

9. Henri R. 2005. Actualité sur la ménopause. Donnée de la médecine factuelle. EMC Endocrinologie. 2(1): 90-101.

10. INS. 2015. Recensement général de la population et des habitations 2014, données socio- démographiques des localités. Institut National de Statistiques : Abidjan.

11. MSLS et INS et ICF International. 2013. Ministère de la Santé et de la Lutte Contre le Sida (MSLS) et 1'Institut National de la Statistique (INS) et ICF International, 2013. Enquête Démographique et de Santé et à Indicateurs Multiples de Côte d'Ivoire 2011-2012 : Rapport de synthèse. Calverton, Maryland, USA : MSLS, INS et ICF International.

12. Kidik P., Ngene J., Ngoule C., Mvogo O., Ndjib R., Dibong S.D., Mpondo Mpondo E. 2015. Caractérisation des plantes médicinales à flavonoïdes des marchés de Douala (Cameroun). International Journal of Biological and Chemical Sciences 9(3): 1494 - 1516

13. Kouamé N.M.T., Gnahoua G.M., Kouassi K.E., Traoré D. 2008. Plantes alimentaires spontanées de la région du Fromager (CentreOuest de la Côte d'Ivoire) : flore, habitats et organes consommés. Sciences \& Nature, 1(5): 61-70.

14. Lagou S.M.L., Tra Bi F.H., Yao K., Bakayoko A., Koné M.W. 2016. Fistules obstétricales dans le district d'Abidjan, Côte d'Ivoire : niveau de connaissance et plantes utilisées traditionnellement dans le 
traitement. International Journal of Biological and Chemical Sciences 10(3): 1273-128.

15. Lapcik O. 2007. Isoflavonoids in non-leguminous taxa: a rarity or a rule? Phytochemistry 68: 2909-2916.

16. Lecerf J.M. 2007. Phytoestrogènes et os : de nouvelles données. Cahiers de nutrition et de diététique. 42: 207-217.

17. Lopès P., Florence T. 2004. Guide pratique de la ménopause, collection Mediguide. Edition Masson. Paris (France), 197 p.

18. Loukid M., Hilali M.K., Bernis C. 2007. Âge à la ménopause naturelle à Marrakech (Maroc) et prévalence des symptômes du climatère. Bulletins et mémoires de la Société d'Anthropologie de Paris. 19: 1-2.

19. Luoto R. 2009. Bouffées de chaleur et qualité de vie pendant la ménopause. BMC Womens Health. 9: 13.

20. Malan F.D., Neuba R.F.D, Kouakou L.K. 2015. Medicinal plants and traditional healing practices in ehotile people, around the aby lagoon (eastern littoral of Côte d'Ivoire). Journal of Ethnobiology and Ethnomedecine. 11: 2.

21. Massé R. 1995. Culture et santé publique. Les contributions de l'anthropologie à la prévention et à la promotion de la santé. Paris, Gaëtan Morin.

22. Mbarga J. 2014. Regards de Suissesses et de Camerounaises citadines sur la ménopause : dépasser les dichotomies binaires », Anthropologie \& Santé [En ligne], 8 |2014, mis en ligne le 31 mai 2014, consulté le 01 mai 2017. URL : http://anthropologiesante.revues.org/1396 ; DOI : 10.4000/anthropologiesante. 1396

23. N'guessan K., Zihiri N., Boraud N.K.M. 2010. Etude ethnopharmacologique des plantes utilisées pour faciliter l'accouchement, en pays Abbey et Krobou, au Sud de la Côte d'Ivoire. International Journal of Biological and Chemical Science. 4(4): 10091010.

24. Palacios S. 2008. Les progrès de la thérapie hormonale substitutive : rendre la ménopause gérable. BMC Womens Health. 8 : 22.

25. Pastor G. 2006. Précis de phytothérapie (Le meilleur de la nature au service de votre santé), Édition Alpen, 100p.

26. Pouamon. 2017. Les pratiques thérapeutiques des femmes ménopausées à Abidjan. Thèse de Doctorat, Université de Cocody Abidjan, UFR Sciences de l'Homme et de la Société, Institut d'ETHNO-SOCIOLOGIE. 343p.

27. Sacks F.M., Lichtenstein A. 2006. Soy protein, isoflavones, and cardiovascular health. American Heart Association Science Advisory for professionals from the Nutrition Committee. 113(7):1034-44. 
28. Sanou S.M., Sali B.B.A., Mabvouna B.R., Douryang M., Teikeu T.V.V., Saah F.M.A., Ovaga E.L.M., Ausseil S.M., Vittorio C., Russo G. 2015. Etude des connaissances, attitudes et pratiques en matière de réintégration sociale des femmes victimes de fistule obstétricale : région de l'Extrême-nord, Cameroun. Pan African Medical Journal. 20(1): 172.

29. Smith J.J, Borgatti S.P. 1998. Salience counts - and so does accuracy: correcting and updating a measure for free-list-item salience. Journal of Linguistic Anthropology. 7(2): 208-209.

30. Thomas F., Renaud F., Benefice E., DE Meeus T., Guegan J.F. 2001. International variability of age at menarche and menopause: patterns and main determinants. Human Biology, 73(2): 271-290.

31. Yue X.H., Abdallah A.M., Xu Z.M. 2010. Distribution of isoflavones and antioxydant activities of soybean cotyledon, coat and germ. Journal of Food Processing and Preservation. 34: 795-806.

32. Zohra S.F. 2007. Le vécu de la ménopause chez la femme Algérienne. Thèse de doctorat. Université Mentouri Constantine. Faculté des Sciences Humaines et des Sciences Sociales. Département de psychologie et des sciences de l'éducation. 267p. 